\title{
Thermophysical Properties of Metastable Helium-3 and Helium-4
}

\author{
R. Balasubramanian ${ }^{1}$, C. Arul $^{2}$ \\ Department of Physics, Arignar Anna Govt. Arts College, Namakkal 637004, Tamilnadu, India
}

\begin{abstract}
A new four-parameter generalized van der Waals equation of state with Peneloux correction in the repulsive and attractive terms has been proposed. This equation of state has been employed to determine the spinodal, thermodynamic limit of superheat and the ideal tensile strength of helium-3 and helium-4. It has been established that helium-3 and helium-4 can be superheated, under rapid heating, up to temperatures $0.82^{T_{C}}$ and $0.84^{T_{C}}$, respectively. Above these temperature limits, helium-3 and helium-4 undergo phase explosion. It has also been established that the helium-3 and helium-4 undergo rupturing at the negative pressures of $-21.0820 P_{E}$, and $25.998^{P}$ respectively.
\end{abstract}

Keywords: Equation of state, Law of corresponding states, Metastable state, Spinodal, Superheat ,Ideal tensile strength, Helium-3 and Helium-4

\section{Introduction}

The knowledge of thermophysical properties of substances is of scientific and technological significance. Reliable data on the thermo physical properties of substances are required for designing various technological processes and also for understanding the nature of substances. The experimental studies on the thermo physical properties of substances, particularly in the high - temperature region, encounter severe difficulties. This is due to the anomalous behavior of substances in the high - temperature region. Thus, arises a necessity for theoretical studies on the thermo physical properties of substances. Statistical mechanics and thermodynamics provide several approaches for the theoretical studies on the thermo physical properties of substances. One of such commonly employed approach is the development of equation of state for substances.

Helium is a monatomic noble gas. It has the lowest melting( $0.95 \mathrm{~K})$ and boiling $(4.222 \mathrm{~K})$ points. It has nine isotopes out of which only two i.e. helium-3 (with 1 neutron) and helium4 (with 2 neutrons) are stable.Helium-3 atoms are fermions and the helium- 4 atoms are bosons. Due to low molar mass, helium has greater thermal conductivity, specific heat and sound speed than any other gas except hydrogen.

Helium has high diffusivity through solids. Helium is the least water-soluble monatomic gas. Refractive index of helium is about 1.At normal ambient temperatures, helium has negative Joule-Thomson coefficient. Joule-Thomson inversion temperature of helium is about 32 to $50 \mathrm{~K}$ at $1 \mathrm{~atm}$. Unlike any other element, helium remains as a liquid down to absolute zero at normal pressure. This is a quantum mechanical effect. Often, it is hard to distinguish solid from liquid helium since the refractive indices of the two phases are nearly the same. Though being inert, helium can form compounds known as excimers, with tungsten, iodine, sulphur and phosphorous when subjected to glow discharge, to electron bombardment or reduced to plasma by other means.
Helium is used as a controlled atmosphere in the growth of $\mathrm{Si}$ and Ge crystals, production of titanium and zirconium, gaschromatography, supersonic wind tunnels, impulse facilities and arc welding. Due to high diffusivity through solids, helium is used as a trace gas to detect leaks in highvacuum equipments and high-pressure containers. Helium is used to displace fuel and to oxidize it in storage tanks. Helium is used to condense hydrogen and oxygen to make rocket fuel. Helium is used as a purge fuel and oxidizer from ground-support equipment in space vehicles. Due to extremely low refractive index, helium is used in solar telescopes. Helium is used in cryogenics to maintain very low temperatures. Helium is used in the study of superconductivity and super fluidity.Due to high thermal conductivity, helium is used as a heat-transfer medium in gas-cooled nuclear reactors. Helium-3has the potential to be used as a fuel in future nuclear fusion power plants. Moreover, Helium-3 has a high absorption cross section for thermal neutron beams and is used as a converter gas in neutron detectors. Considering these facts, the study of the thermo physical properties of helium acquires scientific and technological significance.

In this work, the known two - parameter van der Waals equation of state is generalized by introducing the Peneloux correction in the repulsive and in the attractive terms. The performance characteristics of new fourparameter generalized van der Waals equation of state in describing the high - temperature properties of helium-3 and helium-4, are investigated.

\section{Generalization of Van Der Waals Equation of State}

Many modifications of the known equations of state have been proposed [1-7] to improve the accuracy. These modifications result in the generalization of the known equations of state. There is a need for the generalized equations of state with numerical stability and ability to describe the thermodynamic properties of wide range of technically important substances used in industries. 


\section{International Journal of Science and Research (IJSR) \\ ISSN (Online): 2319-7064}

Index Copernicus Value (2015): 78.96 | Impact Factor (2015): 6.391

In this work, an improvement of the known two parameter van der Waals equation of state is proposed by introducing a third parameter $\mathrm{c}$ in the attractive and repulsive terms. The parameter $\mathrm{c}$ is called the Peneloux correction. Such a generalized van der Waals equation of state for one mole of substance has the form:

$$
P=\frac{R T}{V-b+c}-\frac{a}{(V+c)^{n}}
$$

where $P$ - Pressure, $V$ - Molar volume, $T$ Temperature, $R$ - Universal gas constant, and $a, b, c$ are substance-specific constants.

Differentiating Eq. (1) with respect to $V$, we get

$$
\begin{aligned}
& \left(\frac{\partial P}{\partial V}\right)_{T}=-\frac{R T}{(V-b+c)^{2}}+\frac{n a}{(V+c)^{n+1}} \\
& \left(\frac{\partial^{2} P}{\partial V^{2}}\right)_{T}=\frac{2 R T}{(V-b+c)^{3}}-\frac{n(n+1) a}{(V+c)^{n+2}}
\end{aligned}
$$

The vapor-liquid critical point conditions are

$$
\left(\frac{\partial P}{\partial V}\right)_{T_{c}}=0 \quad ; \quad\left(\frac{\partial^{2} P}{\partial V^{2}}\right)_{T_{c}}=0
$$

From Eq. (2) - (4), we get the critical volume as

$$
\begin{gathered}
V_{c}=N b-c \\
N \equiv \frac{n+\mathbf{1}}{n-\mathbf{1}}
\end{gathered}
$$

Back substitution of Eq (5) into Eq.(2) or Eq. (3), we get the critical temperature as

$$
T_{c}=\frac{4 n a}{R(n-1)^{2} N^{n+1} b^{n-1}}
$$

Substitution Eq. (5) and (6) into Eq.(1), we get the critical pressure as

$$
P_{C}=\frac{a}{N^{n+1} b^{n}}
$$

When Eqs.(5)-(7) are taken into account, the generalized van der Waals equation of state with Peneloux correction gives critical compressibility factor as

$$
Z_{c} \equiv \frac{P_{c} V_{c}}{R T_{c}}=\frac{N b-c}{n(N-1)^{2} b}
$$

The generalized van der Waals equation of state with Peneloux correction may be rewritten in terms of the reduced variables

$$
\begin{gathered}
P^{*}=P / P_{c}, V^{*}=V / V_{c}, T^{*}=T / T_{c} \text { as } \\
P^{*}=\frac{n(N-\mathbf{1})^{\mathbf{2}} b T^{*}}{(N b-c) V^{*}-b+c}-\frac{N^{n+1} b^{n}}{\left[(N b-c) V^{*}+c\right]^{n}}
\end{gathered}
$$

The reduced equation of state given by Eq. (9) represents the three-parameter law of corresponding states with the thermodynamic similarity parameters $b, c$ and $n$.That is, substances obeying the generalized van der Waals equation of state, with the same values of parameter $b, c$ and $n$ are thermodynamically similar. As seen from Eq. (5) and (8), the critical volume and critical compressibility factor depend only on the parameterb,c and $n$ but not on the parameter $a$.On the other hand, Eqs. (6) and (7) show that the critical temperature and the critical pressure depend only on the parameters $a, b$ and $n$ but not on the parameter $c$.

\section{Metastable State of Fluids}

The explosive boiling or phase explosion is a method whereby a superheated metastable liquid undergoes an explosive liquid - vapor phase transition into a stable two - phase state because of a bubbles.

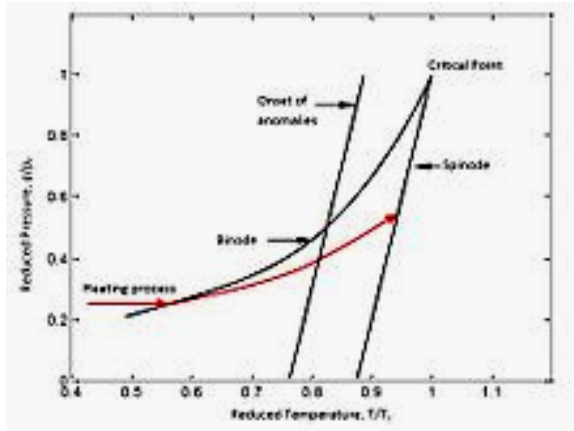

Figure 1: A Typical Phase Diagram of Substances

The phenomenon of explosive boiling was conceptualized by Martynyuk [8] and later advanced by Fucke and Seydel [9]. Fig.1 shows a typical p-T phase diagram of a substance. The explosive boiling can be described by a p-T phase diagram (Fig.1). The binodal line or the coexistence curve is a thermodynamic state where at that specific temperature and pressure, liquid and vapor can coexist. The spinodal line on right is the boundary of absolute instability of a solution to decomposition into multiple phases.

If the heating process is relatively slow, the liquid follows the binodal curve. On the other hand, if the heating process is fast enough that the substance cannot reach binodal curve through heterogeneous boiling, the liquid becomes superheated [10-13] with its temperature above boiling point at a given pressure. System then shifts away from the binodal and thus approaches towards spinodal.

In the absence of bubble - forming nuclei, massive homogeneous nucleation of vapor liquids may be heated to temperatures far above their boiling points. There is, however, an absolute limit of superheat, determined the limit of mechanical stability of fluids, which for a pure substance is $-\left(\frac{\partial P}{\partial V}\right)_{\mathrm{T}}=0$. The limit of superheat is only about $10 \%$ below the critical 


\section{International Journal of Science and Research (IJSR) \\ ISSN (Online): 2319-7064 \\ Index Copernicus Value (2015): 78.96 | Impact Factor (2015): 6.391}

temperature of many substances. The limit of superheating is an important property of liquids, which is relevant for the design and safety assessment of processes involving pressurized liquids. The limit of superheat of a liquid represents the deepest possible penetration of a liquid in the domain of metastable states. It is therefore important to accurately predict the conditions under which liquids can undergo vapor explosive boiling.

The ability to rigorously define in mathematical terms the limit of stability of a liquid supports the belief that it is a fundamental thermodynamic property. It may be considered a kind of "critical point" of a liquid corresponding to each pressure.

\subsection{Spinodal}

The spinodol is therefore, defined by the condition:

$$
-\left(\frac{\partial P}{\partial V}\right)_{T}=\mathbf{0}
$$

Applying the condition given by Eq.(10) to Eq.(9), we get the equation of spinodal in $T^{*}, V^{*}$ coordinates :

$$
T_{s}^{*}=\frac{N^{n+1}\left[\left(N-\frac{c}{b}\right) V^{*}+\frac{c}{b}-1\right]^{2}}{(N-1)^{2}\left[\left(N-\frac{c}{b}\right) V^{*}+\frac{c}{b}\right]^{n+1}}
$$

Substituting Eq. (11) into Eq. (9), we get the equation of spinodal in $P^{*}, V^{*}$ coordinates :

$$
P_{s}^{*}=\frac{N^{n+1}}{\left[\left(N-\frac{c}{b}\right) V^{*}+\frac{c}{b}\right]^{n}}\left[\frac{n\left[\left(N-\frac{c}{b}\right) V^{*}+\frac{c}{b}-1\right]}{\left[\left(N-\frac{c}{b}\right) V^{*}+\frac{c}{b}\right]}-1\right]
$$

\subsection{Thermodynamic Limit of Superheat}

With decrease in pressure, the superheat of substances increases. The thermodynamic limit of superheat is attained at

$$
P=\mathbf{0}
$$

Applying the condition given by Eq.(13) to Eq.(9), we get the reduced volume of the fluid at the thermodynamic limit of superheat as

$$
V_{s, p=0}^{*}=\frac{n b-(n-1) c}{(n+1) b-(n-1) c}
$$

Eq.(14) may be rewritten as

$$
V_{s_{x} P=0}=\left[\frac{n b-(n-1) c}{(n+1) b-(n-1) c}\right] V_{c}
$$

That is, the reduced volume of the fluid at the thermodynamic limit of superheat depends on parameters $b, c$ and $n$ of the generalized van der Waals equation of state with Peneloux correction. Substituting
Eq.(14) into Eq.(11), we get the thermodynamic limit of superheat as

$$
T_{s, p=0}^{*}=\frac{\mathbf{1}}{\mathbf{4}}\left(\frac{n+\mathbf{1}}{n}\right)^{n+\mathbf{1}}
$$

Eq.(16) may be rewritten as

$$
T_{S_{x} P=0}=\frac{1}{4}\left(\frac{n+1}{n}\right)^{n+1} T_{C}
$$

That is, thermodynamic limit of superheat of substances depends only on the parameter $n$ but not on the parameters $a, b$ and $c$ of the generalized of van der Waals equation of state with Peneloux correction.

\subsection{Ideal Tensile Strength}

The ideal tensile strength is the theoretical limiting negative pressure,excerted from all sides, at which a liquid breaks down. It is a temperature-dependent characteristic property of fluids.

$$
\begin{aligned}
& \text { At } T=0 \text {,Eq.(11) gives } \\
& \qquad V_{s, T=0}^{*}=\frac{(n-1)(b-c)}{(n+1) b-(n-1) c}
\end{aligned}
$$

Eq.(18) may be rewritten as

$$
V_{s, T=0}=\left[\frac{(n-1)(b-c)}{(n+1) b-(n-1) c}\right] V_{c}
$$

Substituting Eq.(19) into Eq.(12),we get the ideal tensile strength of fluids as

$$
P_{s_{x} T=0}^{*}=-\left(\frac{n+1}{n-1}\right)^{n+1}
$$

Eq.(20) may be rewritten as

$$
P_{s, T=0}=-\left(\frac{n+1}{n-1}\right)^{n+1} P_{C}
$$

As seen,the reduced ideal strength depends only the parameter $n$ and not on the parameters $a, b, c$.At this point, the reduced volume depends only the parameters $b, c n$ and not on the parameter $a$.

\section{Calculations and Analysis}

The parameters $a, b$ and $c$ for helium-3 and helium-4 are determined through the Eqs.(5)-(8) using experimental data[14] on critical-point parameters along with the values of $n$.The obtained values of $a, b$ and $c$ are presented in Table1.

Table 1: Equation-of -State Parameters for Helium-3 and Helium-4

\begin{tabular}{|c|c|c|c|c|}
\hline Noble gas & $\begin{array}{c}a \\
10^{-6} \mathrm{~Pa} .\left(\mathrm{m}^{3} / \mathrm{mol}\right)^{\mathrm{n}}\end{array}$ & $\begin{array}{c}b 10^{-6} \\
\mathrm{~m}^{3} / \mathrm{mol}\end{array}$ & $\begin{array}{c}c 10^{-6} \\
\mathrm{~m}^{3} / \mathrm{mol}\end{array}$ & $n$ \\
\hline Helium-3 & 163.765 & 45.313 & 40.238 & 2.343 \\
\hline Helium-4 & 2391.800 & 25.269 & 16.388 & 2.0433 \\
\hline
\end{tabular}

Considering the values of $n$ (Table1) for helium-3 and helium-4,the spinodal is determined by Eqs.(11)and (12).The obtained spinodal-parameters are presented in Tables2\&3.These spinodal-parameters define the stability boundary of helium-3 and helium-4 in the phase diagram.

\section{Volume 6 Issue 1, January 2017




\section{International Journal of Science and Research (IJSR) \\ ISSN (Online): 2319-7064}

Index Copernicus Value (2015): 78.96 | Impact Factor (2015): 6.391

Table 2: Spinodal of Helium-3

\begin{tabular}{|c|c|c|}
\hline $\boldsymbol{V}^{*}$ & $\boldsymbol{T}_{\boldsymbol{s}}^{*}$ & $\boldsymbol{P}_{\boldsymbol{s}}^{*}$ \\
\hline 0.100 & 0.0188 & -16.860 \\
\hline 0.200 & 0.0733 & -10.389 \\
\hline 0.300 & 0.453 & -3.723 \\
\hline 0.400 & 0.642 & -1.488 \\
\hline 0.500 & 0.782 & -0.273 \\
\hline 0.600 & 0.878 & 0.379 \\
\hline 0.700 & 0.940 & 0.726 \\
\hline 0.800 & 0.976 & 0.903 \\
\hline 0.900 & 0.995 & 0.980 \\
\hline 1.000 & 1.000 & 1.000 \\
\hline
\end{tabular}

Table 3: Spinodal of Helium-4

\begin{tabular}{|c|c|c|}
\hline $\boldsymbol{V}^{*}$ & $\boldsymbol{T}_{\boldsymbol{s}}^{*}$ & $\boldsymbol{P}_{\boldsymbol{s}}^{*}$ \\
\hline 0.100 & 0.163 & -43.929 \\
\hline 0.200 & 0.0551 & -17.216 \\
\hline 0.300 & 0.325 & -7.185 \\
\hline 0.400 & 0.569 & -2.843 \\
\hline 0.500 & 0.746 & -0.819 \\
\hline 0.600 & 0.862 & -0.166 \\
\hline 0.700 & 0.933 & 0.652 \\
\hline 0.800 & 0.975 & 0.881 \\
\hline 0.900 & 0.995 & 0.977 \\
\hline 1.000 & 1.000 & 1.000 \\
\hline
\end{tabular}

The parameters of the thermodynamic limit of superheat for helium-3 and helium-4 are determined through the Eqs.(14)-(17) using the values of the parameters $b, c$ and $n$ (Table1) along with experimental data[14] on critical point. The obtained values are presented in Table4.

Table 4: Thermodynamic Limit of Superheat of Helium-3 and Helium-4

\begin{tabular}{|c|c|c|c|c|}
\hline Noble gas & $\boldsymbol{V}_{s, p=0}^{*}$ & $\boldsymbol{T}_{s, p=0}^{*}$ & $\begin{array}{c}\boldsymbol{V}_{s, P=0} \\
10^{-6} \mathrm{~m}^{3} / \mathrm{mol}\end{array}$ & $\begin{array}{c}\boldsymbol{T}_{s, P}=0 \\
\mathrm{~K}\end{array}$ \\
\hline Helium-3 & 0.535 & 0.820 & 38.788 & 2.714 \\
\hline Helium-4 & 0.578 & 0.840 & 33.119 & 4.368 \\
\hline
\end{tabular}

Below the thermodynamic limit of superheat, heterogeneous nucleation will prevail. And, above the thermodynamic limit of superheat, homogeneous nucleation will prevail resulting in the explosive boiling. The ideal tensile strength of helium3 and helium-4 is determined through Eqs.(18)- (21) using the values of the parameters $n$ (Table1). The obtained values are presented in Table5.

Table 5: Ideal Tensile Strength of Helium-3 and Helium-4

\begin{tabular}{|c|c|c|c|c|}
\hline Noble gas & $\boldsymbol{V}_{s, T=0}^{*}$ & $\boldsymbol{P}_{s, T=0}^{*}$ & $\begin{array}{c}\boldsymbol{V}_{s,}^{*}, T=0 \\
10^{-6} \mathrm{~m}^{3} / \mathrm{mol}\end{array}$ & $\begin{array}{c}\boldsymbol{P}_{s, T=0} \\
\mathrm{MPa}\end{array}$ \\
\hline Helium-3 & 0.0670 & -21.0820 & 4.858 & -2.467 \\
\hline Helium-4 & 0.155 & -25.998 & 8.882 & -5.928 \\
\hline
\end{tabular}

\section{Conclusion}

A new four- parameter generalized van der Waals equation of state with Peneloux correction has been employed to calculate the spinodal, thermodynamic limit of superheat and the ideal tensile strength of helium-3 and helium-4. The obtained spinodal-parameters of helium-3 and helium-4 determine the stability boundary in the phase diagram. The knowledge of the stability boundary is essential for the study of helium-3 and helium-4 in the metastable state. It has been established that helium-3 and helium-4 can be superheated, under rapid heating, up to temperatures $0.82^{T_{c}}$ and $0.84 T_{c}$, respectively. Above these temperature limits, helium-3 and helium-4 undergo phase explosion. This fact has to be taken into account when helium-3 and helium4 are subjected to rapid heating. It has also been established that the helium-3 and helium-4 undergo rupturing at the negative pressures of $-21.0820 P_{e}$ and $-25.998 P_{e}$, respectively. This fact is to be taken into account when helium-3 and helium-4 are subjected to high negative pressures.

\section{References}

[1] Martynyuk M M, "Generalized van der Waals Equation of State for Liquids and Gases",Zh.Fiz.Khim.,65,1716$1717,1991$.

[2] Martynyuk M M and Balasubramanian R, "Equation of State for Liquid Alkali Metals:Binodal", Int.J.Thermophys.,16,533-543,1995.

[3] LguchiM,MachidaH,SatoY,Richard L and Smith Jr., " $\varepsilon$ "-Modified Sanchez-Lacombe Equation of State", Asia-Pacific J.Chem.Eng.,7,95-100,2012.

[4] Alexandrov I et al., "Hemholtz Energy based Empirical Model",Int.J.Thermophys., 34,1865 - 1905, 2013.

[5] Balasubaramanian R, Gunavathi.K, Jegan.R and Roobanguru D," A study on the generalisation of equations of state for liquids and gases", Open Journal of Modern Physics, 1, 54-60,2014.

[6] Balasubramanian R and Kamala R, "A New Threeparameter Generalized Berthelot Equation of State for Hydrocarbons: Superheat", OpenSci.J Modern.Phys.3,14,2016.

[7] Balasubramanian R and Menaka S, "A Study on the Thermodynamic Limit of Superheat of Refractory Metals",Int.J.Sci.Res.,5,634-636,2016.

[8] Martynyuk, M. M. "Phase Explosion of a Metastable Fluid". Combustion, Explosion, and Shock Waves 13 (2): 178-191,1977.

[9] Seydel, U; Fucke, W, "Experimental Determination of Critical Data of Liquid Molybdenum". Journal of Physics F: Metal Physics 8 (7): L157-L161,1978.

[10] Martynyk M.M, "Superheating of Solid and Liquid Metals in the Process of Pulse Heating",Thermochim.Acta ,206,55-60,1992.

[11] Balasubramanian.R. "Superheating of Liquid Alkali Metals "Int.J.Thermophys, 27,1494-1500,2006.

[12] Balasubramanian.R. "Correlations of Attainable Superheat of Fluid AlkaliMetals",J.Nuclear Materials,366,272-276,2007.

[13] Balasubramanian.R. "Correlations of Supercritical Temperature of Fluid AlkaliMetals",Asia-Pacific J.Chem. Eng.,3,90-94,2008.

[14] Yaws C.L, "Thermophysical Properties of Chemicals and Hydrocarbons", $2^{\text {nd }}$ Edition, Gulf Professional Publishing,126-128,2014.

\section{Volume 6 Issue 1, January 2017 www.ijsr.net}




\section{Author Profile}

Dr. R. Balasubramanianis an Assistant Professor of Physics at the Arignar Anna Government Arts College, Namakkal, India. He has 24 years of postdoctoral research and teaching experience. He did his Doctorate at the Russian Peoples' Friendship University, Moscow, Russia under the supervision of renowned Professor M.M.Martynyuk who conceptualized the phenomenon of Explosive Boiling of liquids. His research interests include Phase transitions, Critical phenomena and Equations of state.

C. Arul is a research scholar in the Department of Physics at the Arignar Anna Government Arts College, Namakkal, India. 